\title{
Sex differences in 10-year ischemic cardiovascular disease risk prediction in Chinese patients with prediabetes and type 2 diabetes
}

Lihong Yang ${ }^{1,2,3}$, Anne F. Fish ${ }^{4}$, Yuanyuan Zhu ${ }^{5}$, Xiaodan Yuan ${ }^{1,2}$, Jianing Li ${ }^{1,2}$, Xiaoyun Wang ${ }^{6}$, Li Yuan ${ }^{7}$, Zhumin $\mathrm{Jia}^{8}$, Chao Liu ${ }^{1,2}$, Yunchen $\mathrm{Xu}^{9^{*}}$ and Qingqing Lou ${ }^{1,2^{*}}$ (D)

\begin{abstract}
Background: Cardiovascular disease has become a serious public health problem in recent years in China. The aim of the study was to examine sex differences in cardiovascular risk factors and 10-year ischemic cardiovascular disease (ICVD) risk in Chinese patients with prediabetes (PreDM) and type 2 diabetes mellitus (T2DM).

Methods: This was a multi-site retrospective case-control study conducted from April-November 2016 using an electronic medical record database, involving 217 PreDM and 900 T2DM patients admitted to endocrinology units in four hospitals in China. CVD risk was estimated using the Chinese 10-year ICVD risk model. The differences in 10-year absolute ICVD risk according to PreDM, T2DM $<1$ year, T2DM 1-5 years or T2DM $\geq 5$ years and sex were analyzed using ANOVA.
\end{abstract}

Results: When compared to PreDM females, males with PreDM had significantly higher 10-year ICVD risk In contrast, the opposite pattern of 10-year ICVD risk was observed in T2DM; males had significantly lower 10-year ICVD risk. Moreover, compared to T2DM females, males with T2DM had a lower proportion $s$ with moderate or greater ICVD risk $(p<0.001)$. When compared to PreDM males, males with T2DM $<1$ year, and with T2DM 1-5 years had no difference in 10-year ICVD risk, but had higher ICVD risk with T2DM $\geq 5$ years $(p<0.05)$. Compared to PreDM females, females with T2DM in all subgroups had higher ICVD risk $(p<0.05)$. Among those with T2DM, hypertension rates of awareness, treatment and control were $78.60 \%, 65.38 \%$ and $31.10 \%$, respectively; hyperlipidemia rates of awareness, treatment and control were lower (29.15\%, 8.30\% and 3.47\%, respectively). Females with T2DM had higher prevalence, awareness and treatment of hypertension and hyperlipidemia than males with T2DM $(p<0.001)$.

Conclusions: There is a greater need for cardiovascular risk reduction programs for females with T2DM at diagnosis. Given the low numbers for awareness, treatment and control of hypertension and hyperlipidemia in both males and females, significant resources focused on them must be expended, specifically improving regular assessment of blood pressure and blood lipids. Strengthening the management of chronic diseases through adherence to evidence-based guidelines to enhance clinical treatment may reduce 10-year ICVD in patients with T2DM in China.

Keywords: Cardiovascular disease, Risk assessment, Duration of diabetes, Prediabetes, Case control studies

\footnotetext{
* Correspondence: 1491400746@qq.com; lqqmedicine@163.com

${ }^{9}$ Hainan People's Hospital-Longhua, \#8 Longhua Rd, Longhua District Hainan, Haikou 570102, China

${ }^{1}$ Department of Health Education, Affiliated Hospital of Integrated Traditional Chinese and Western Medicine, Nanjing University of Chinese Medicine, \#100 Hongshan Road, Qi xia District, Nanjing, Jiangsu Province 210028, China Full list of author information is available at the end of the article
}

(c) The Author(s). 2019 Open Access This article is distributed under the terms of the Creative Commons Attribution 4.0 International License (http://creativecommons.org/licenses/by/4.0/), which permits unrestricted use, distribution, and reproduction in any medium, provided you give appropriate credit to the original author(s) and the source, provide a link to the Creative Commons license, and indicate if changes were made. The Creative Commons Public Domain Dedication waiver (http://creativecommons.org/publicdomain/zero/1.0/) applies to the data made available in this article, unless otherwise stated. 


\section{Background}

In 2017, the International Diabetes Federation reported that China had the highest number of adults (20-79 years) with diabetes mellitus. Its Diabetes Society refers to China as the global epicenter of the diabetes epidemic. About 114.4 million Chinese adults have diabetes, and its prevalence is expected to rise to 119.8 million by 2045 [1]. Overall, the prevalence of type 2 diabetes mellitus (T2DM) is similar in women and men in China, the prevalence among men was $12.1 \%$ and among women was $11.0 \%$ [2]. Prediabetes (PreDM) has dramatically increased in China, rising from $15.5 \%$ in 2007 to $50.1 \%$ in 2010 [2, 3]. According to the 20-year China Da Qing Diabetes Prevention Study [4], 93\% of persons with PreDM will progress to overt T2DM in 20 years, imposing a large health and economic burden on China. In addition, PreDM is also an important risk factor for the development of cardiovascular disease [5].

Recently, ischemic cardiovascular disease (ICVD) is increasing in China, although it appears to have fallen over the past 2 decades in the US and Europe [6]. In 2013, the age-standardized mortality rate for ischemic heart disease increased by $2.6 \%$ and for stroke increased by $28.8 \%$ [7]. About 2 out of every 5 deaths is from cardiovascular disease, the leading cause of death in China [8]. Some evidence suggests that T2DM confers a stronger excess risk of cardiovascular diseases in women than in men $[9,10]$, yet a National Health and Nutrition Examination Survey (NHANES) [11] in the US showed that women in the midlife years have historically been at a lower risk for overall vascular events than similarly aged men. Therefore, examining sex differences in ICVD risk and preventing ICVD in China in those already diagnosed with T2DM are important goals in diabetes care.

A Chinese task group of the national Fifteen Project developed sex-specific optimal 10-year risk prediction models in the China MUCA study cohort II [12]. Distinct from models developed in Europe, the Chinese 10year ICVD risk model estimates total ICVD risk (both coronary heart disease and stroke). It is appropriate for Chinese racial and ethnic groups and has been incorporated into current Chinese guidelines for cardiovascular disease prevention. Although several studies have used the 10-year ICVD risk in Chinese adults, its prediction of sex differences in those with PreDM and T2DM has not been reported [13-15]. Hence, in the current study, we examined CVD risk factors and used an established ICVD tool to examine the 10-year ICVD risk in male and female Chinese adults with PreDM and T2DM. This study may identify groups at higher risk, the consequence of which is that health care providers could use this information to deliver lifestyle modification programs or pharmacological interventions within diabetes care that target the most vulnerable groups.

\section{Methods}

\section{Design and sample selection}

A retrospective case-control study design was used. Electronic medical records were obtained from all patients admitted to endocrinology units in four hospitals, one in each province (Jiangsu, Henan, Shanxi and Sichuan) from April 2016 to November 2016. The majority of the inpatients in the study were admitted for blood glucose adjustment or for annual chronic complication screening not for illness. This practice is very different from th at in western countries because insurance provides more generous reimbursement for inpatient care in China, which incentivizes patients to get hospitalized for even minor health conditions. Hospital discharge data obtained from electronic medical records included demographics, medical history (diagnoses), diabetes status, laboratory tests, drug treatments and blood glucose records. The Scientific Research Committees and Ethics Committees of the four hospitals approved the study.

There were 217 patients with PreDM in the data base. Age and sex -matched T2DM patient records $(n=900)$ were obtained from the database of the four centers for the calculation of 10-year ICVD risk. We included in the analysis those who were $\geq 35$ years of age and had a diagnosis of PreDM or T2DM confirmed using a screening 2 -h oral glucose tolerance test (OGTT). Participants were diagnosed with PreDM according to American Diabetes Association criteria: impaired fasting glucose (5.6 to $6.9 \mathrm{mmol} / \mathrm{L}$ [100 to $125 \mathrm{mg}$ / $\mathrm{dL}]$ ) and/or impaired oral glucose tolerance (7.8 to 11.1 $\mathrm{mmol} / \mathrm{L}$ [140 to $199 \mathrm{mg} / \mathrm{dL}]$. Participants were diagnosed with T2DM according to Guidelines for the Prevention and Treatment of T2DM in China (2013 Edition) [16], with typical symptoms of diabetes, random plasma glucose $\geq 11.1$ $\mathrm{mmol} / \mathrm{L}$, or fasting plasma glucose (FPG) $\geq 7.0 \mathrm{mmol} / \mathrm{L}$, or 2-h plasma glucose level of $\geq 200 \mathrm{mg} / \mathrm{dL}(11.1 \mathrm{mmol} / \mathrm{L})$ during a 75-g OGTT or hemoglobin A1c (HbA1c) level $\geq 6.5 \%$. Exclusion criteria were pregnancy or lactation, liver or kidney dysfunction, malignant tumor, mental illness, or diagnosis of coronary heart disease or ischemic stroke.

\section{Collection of clinical and laboratory parameters}

Participants underwent anthropometric measurements, questionnaires and blood drawing at baseline. Smoking was defined as daily consumption of more than one cigarette/day for $>1$ year [17]. Weight $(\mathrm{kg})$ and height $(\mathrm{cm})$ were determined using a standard hospital balance scale and a metal ruler; participants wore light clothing and no shoes. Waist circumference was measured at the level of the umbilicus with the patient standing and breathing normally, measured twice and averaged. BMI was calculated as body weight $(\mathrm{kg})$ divided by height $\left(\mathrm{m}^{2}\right)$. Overweight was defined using the Asian standard as BMI of $24 \mathrm{~kg} / \mathrm{m}^{2}$ or greater [18].

Systolic and diastolic blood pressure (SBP, DBP) were taken on the dominant arm in a sitting position using a 
standard manual sphygmomanometer and an adult size cuff [19]. Hypertension was defined as the absence of antihypertensive drugs and SBP $\geq 140 \mathrm{mmHg}$ and/or DBP $\geq 90 \mathrm{mmHg}$ on three different days or by a previous diagnosis of hypertension with current antihypertensive medication [20]. Awareness of hypertension was defined as a participant's self-report of diagnosed high BP. Treated hypertension was defined as antihypertensive drugs being used currently, and controlled hypertension was defined as a participant's report of antihypertensive treatment together with a clinical measurement of $\mathrm{SBP}<140 \mathrm{mmHg}$ and $\mathrm{DBP}<90 \mathrm{mmHg}$ [21].

Total cholesterol (TC), triglycerides (TG), HDL and low-density lipoprotein cholesterol (LDL) were measured after a 10-h fast, using a standardized and reliable method, an automatic biochemical analyzer. Hyperlipidemia was defined as TC $>5.18 \mathrm{mmol} / \mathrm{L}$ or/and TG > $1.70 \mathrm{mmol} / \mathrm{L}$. [22] Awareness of hyperlipidemia was defined as a self-report of any prior diagnosis of hyperlipidemia by a medical doctor. Treatment of hyperlipidemia was defined as use of pharmacological treatment to manage hyperlipidemia. Participants were considered to have controlled hyperlipidemia if the TC was $<5.18$ $\mathrm{mmol} / \mathrm{L}$ and TG was $<1.70 \mathrm{mmol} / \mathrm{L}$ after treatment.

FPG and 2-h plasma glucose (2hPG) concentrations were measured by glucose oxidase methods. HbA1c was measured using the high performance liquid chromatography method, and fasting serum insulin (FSI) concentration was measured using an electrochemiluminescence assay. Homeostatic model assessment of $\beta$-cell function $($ HOMA- $\beta$ ) was calculated as HOMA- $\beta=20 \times$ FSI $/$ FPG3.5). Homeostatic Model Assessment of Insulin Resistance was calculated as HOMA-IR $=(\mathrm{FPG} \times \mathrm{FSI}) / 22.5[23]$.

\section{Calculation of absolute ICVD risk engine based on clinical and biochemical characteristics}

The 10-year ICVD risk scores of participants were evaluated using the ICVD risk engine [12]. The items included: records of sex (male or female), age ( $\geq 35$ years), BMI, current smoking status (no or yes), SBP, TC and diabetes [16]. A risk value was calculated using these seven indices to get an absolute 10-year ICVD risk (Appendix). According to the risk value, participants were divided into extremely high risk $(\geq 40 \%)$, high risk (20-40\%), moderate risk (10-20\%), low risk (5-10\%) and extremely low risk $(<5 \%)$ [24]. Therefore, the commonly used cut-off value of $>10 \%$ indicates moderate or greater risk [24].

\section{Statistical analysis}

Epidata 3.1 and SPSS22.0 were used. Continuous variables are presented as the mean $\pm S D$, whereas categorical variables are presented as frequencies and percentages. Data were tested for normality of distribution by the Kolmogorow-Smirnow test. If data were not normally distributed, the respective nonparametric test was used. Sex differences between baseline characteristics of those with PreDM and T2DM were analyzed using t-tests for independent groups or a non-parametric test and chi-square for categorical variables (smoking and >10\% ICVD risk). We calculated the 10-year ICVD risk of each individual using the risk engine technique. The differences in 10year absolute ICVD risk according to PreDM, T2DM $<1$ year, T2DM $1-5$ years or T2DM $\geq 5$ years and sex were analyzed using ANOVA. Differences in prevalence, awareness, treatment and control for hypertension and hyperlipidemia in males or females with T2DM were analyzed using chi-square. A $p$ value of $\leq .05$ was considered statistically significant.

\section{Results}

\section{Sample characteristics}

Baseline clinical and biochemical characteristics are presented (Table 1). PreDM patients included 77 males and 140 females and those with T2DM included 300 males and 600 females. When compared to PreDM females, males with PreDM had significantly higher 10-year ICVD risk. In contrast, the opposite pattern of 10-year ICVD risk was observed in T2DM; males had significantly lower 10-year ICVD risk. Also, compared to T2DM females, males with T2DM had a lower proportion with moderate or greater ICVD risk $(\mathrm{p}<0.001)$.

\section{0-year ICVD risk in male and female patients with PreDM or T2DM}

We divided the T2DM patients into 3 subgroups according to their diabetes duration: T2DM $<1$ year, T2DM $1-5$ years or T2DM $\geq 5$ years. The pattern of 10 -year ICVD risk across PreDM and T2DM was dissimilar in males and females (Fig. 1 and Fig. 2). When compared to PreDM males, males with T2DM $<1$ year, and with T2DM $1-5$ years had no difference in 10-year ICVD risk, but had higher ICVD risk with T2DM $\geq 5$ years $(p<0.05)$. Compared to PreDM females, females with T2DM in all subgroups had higher ICVD risk $(p<0.05)$. These findings indicate different cut-off points for 10 -year ICVD risk in male and female patients.

\section{Prevalence, awareness, treatment and control of hypertension and hyperlipidemia in male and female Chinese with T2DM}

The awareness, treatment and control of hypertension and hyperlipidemia overall $(n=900)$ were: $78.60 \%$ were aware of the hypertension diagnosis, $65.38 \%$ were receiving treatment and $31.10 \%$ had hypertension controlled, whereas those for hyperlipidemia were lower $(29.15 \%, 8.30 \%$ and $3.47 \%$, respectively). These outcomes in Chinese with T2DM are presented according to sex in Table 2. Females with T2DM had a significantly higher prevalence, awareness, treatment and control of hypertension and also prevalence, awareness and treatment of hyperlipidemia than males with T2DM . The numbers for awareness, treatment and control of hypertension and hyperlipidemia were very low in both males and females. 
Table 1 Baseline clinical and biochemical characteristics among Chinese patients with PreDM or T2DM

\begin{tabular}{|c|c|c|c|c|c|c|}
\hline \multirow[t]{2}{*}{ Variable } & \multicolumn{2}{|l|}{ PreDM } & \multirow[b]{2}{*}{$p$ value } & \multicolumn{2}{|l|}{ T2DM } & \multirow{2}{*}{$\begin{array}{l}p \\
\text { value }\end{array}$} \\
\hline & $\begin{array}{l}\text { Male } \\
(n=77)\end{array}$ & $\begin{array}{l}\text { Female } \\
(n=140)\end{array}$ & & $\begin{array}{l}\text { Male } \\
(n=300)\end{array}$ & $\begin{array}{l}\text { Female } \\
(n=600)\end{array}$ & \\
\hline Age (years) & $61.55 \pm 5.18$ & $61.36 \pm 5.36$ & 0.127 & $61.13 \pm 4.46$ & $61.51 \pm 6.53$ & 0.082 \\
\hline BMI $\left(\mathrm{kg} / \mathrm{m}^{2}\right)$ & $24.89 \pm 2.67$ & $24.85 \pm 3.20$ & 0.299 & $24.68 \pm 3.47$ & $25.12 \pm 3.76$ & 0.148 \\
\hline WC (cm) & $87.75 \pm 8.44$ & $85.07 \pm 9.02$ & 0.015 & $89.49 \pm 9.05$ & $84.92 \pm 8.67$ & $<0.001$ \\
\hline Smoking (\%) & $31(40.26)$ & $1(0.71)$ & $<0.001$ & 128(42.67\%) & $11(1.83 \%)$ & $<0.001$ \\
\hline $\mathrm{SBP}(\mathrm{mmHg})$ & $134.91 \pm 16.82$ & $132.48 \pm 16.28$ & 0.142 & $135.29 \pm 20.05$ & $135.23 \pm 18.45$ & 0.830 \\
\hline $\mathrm{DBP}(\mathrm{mmHg})$ & $79.35 \pm 11.93$ & $79.70 \pm 10.82$ & 0.977 & $80.31 \pm 11.09$ & $79.09 \pm 9.99$ & 0.146 \\
\hline TC (mmol/L) & $5.03 \pm 0.89$ & $5.24 \pm 1.00$ & 0.087 & $4.33 \pm 1.17$ & $4.68 \pm 1.24$ & $<0.001$ \\
\hline TG (mmol/L) & $1.78 \pm 0.94$ & $1.85 \pm 1.02$ & 0.675 & $1.83 \pm 1.30$ & $1.95 \pm 1.40$ & 0.102 \\
\hline $\mathrm{HDL}(\mathrm{mmol} / \mathrm{L})$ & $1.40 \pm 0.39$ & $1.51 \pm 0.37$ & 0.072 & $1.09 \pm 0.31$ & $1.24 \pm 0.54$ & $<0.001$ \\
\hline LDL (mmol/L) & $3.00 \pm 0.78$ & $3.10 \pm 0.72$ & 0.367 & $2.78 \pm 0.87$ & $2.92 \pm 1.20$ & 0.072 \\
\hline FPG (mmol/L) & $5.89 \pm 0.58$ & $5.86 \pm 0.59$ & 0.890 & $8.02 \pm 2.57$ & $8.29 \pm 2.92$ & 0.305 \\
\hline $2 \mathrm{hPG}(\mathrm{mmol} / \mathrm{L})$ & $7.96 \pm 1.88$ & $7.73 \pm 1.54$ & 0.257 & $16.01 \pm 5.01$ & $16.52 \pm 4.98$ & 0.216 \\
\hline $\mathrm{HbA1c}(\%)$ & $5.99 \pm 0.36$ & $5.94 \pm 0.34$ & 0.328 & $9.23 \pm 2.51$ & $9.10 \pm 2.49$ & 0.402 \\
\hline $\mathrm{FSI}(\mu \mathrm{IU} / \mathrm{ml})$ & $10.39 \pm 4.58$ & $11.46 \pm 4.00$ & 0.114 & $8.81 \pm 6.35$ & $10.24 \pm 7.97$ & 0.007 \\
\hline HOMA- $\beta$ & $61.13 \pm 26.97$ & $67.41 \pm 23.56$ & 0.114 & $51.59 \pm 47.94$ & $57.41 \pm 53.22$ & 0.124 \\
\hline HOMA-IR & $2.70 \pm 1.19$ & $3.00 \pm 1.13$ & 0.145 & $3.20 \pm 2.90$ & $3.72 \pm 3.27$ & 0.005 \\
\hline 10-year ICVD risk (\%) & $7.54 \pm 6.71$ & $4.97 \pm 5.20$ & $<0.001$ & $9.64 \pm 9.58$ & $11.77 \pm 10.56$ & 0.014 \\
\hline Moderate or greater ICVD risk $\mathrm{n}(\%)$ & $16(20.78)$ & $23(16.43)$ & 0.424 & $71(23.67)$ & 283(47.17) & $<0.001$ \\
\hline
\end{tabular}

Values are mean \pm SD. BMI, Body mass index; WC, waist circumference; SBP, Systolic blood pressure; DBP, Diastolic blood pressure; TC, Total cholesterol; TG, Triglyceride; HDL, High density lipoproteins; LDL, Low density lipoproteins FPG, Fasting plasma glucose; 2hPG, 2-h plasma glucose; HbA1c, Glycated hemoglobin; FSI, Fasting serum insulin; HOMA- $\beta$, Homeostasis model assessment of beta cell function; HOMA-IR, Homeostasis model assessment of insulin resistance

\section{Discussion}

An interesting aspect of our study is that the 10-year ICVD risk was higher in males with PreDM, whereas there is a definite converse difference in T2DM. A similar finding [25] was observed in a previous studythat showed, at a given glycemic state, the absolute glycemic CVD risk was higher in males than in females. However, this difference narrowed when progressing from normal glycaemia to newly diagnosed diabetes, and the relative risk of CVD was higher in females with DM in comparison with male counterparts. Additionally, males with T2DM compared to females had a significantly lower proportion with a moderate or greater risk category. This is inconsistent with a Caribbean study, which reported a relatively higher proportion of male patients at $>15 \%$ 10-year ICVD risk compared to females $(60.4 \%$ vs $30.6 \%$ ) [26]. This difference in results might be due to different models and algorithms for CVD risk calculation with different risk categories in different races. Our study suggested that Chinese female T2DM patients are at higher 10-year ICVD risk, and great attention should be paid to this finding.

Compared to PreDM, we found that a significantly high 10-year ICVD risk occurred in females with T2DM at $<1$ year, yet this finding was observed later in males with T2DM at $\geq 5$ years. The implication is that earlier intervention programs should be implemented for female patients with T2DM. There are two reasons that may account for the 10-
Fig. 1 Assessment of 10-year ICVD risk in males with PreDM and T2DM.Values are mean $\pm S D .{ }^{*} p \leq 0.05$, compared T2DM to PreDM in male patients 


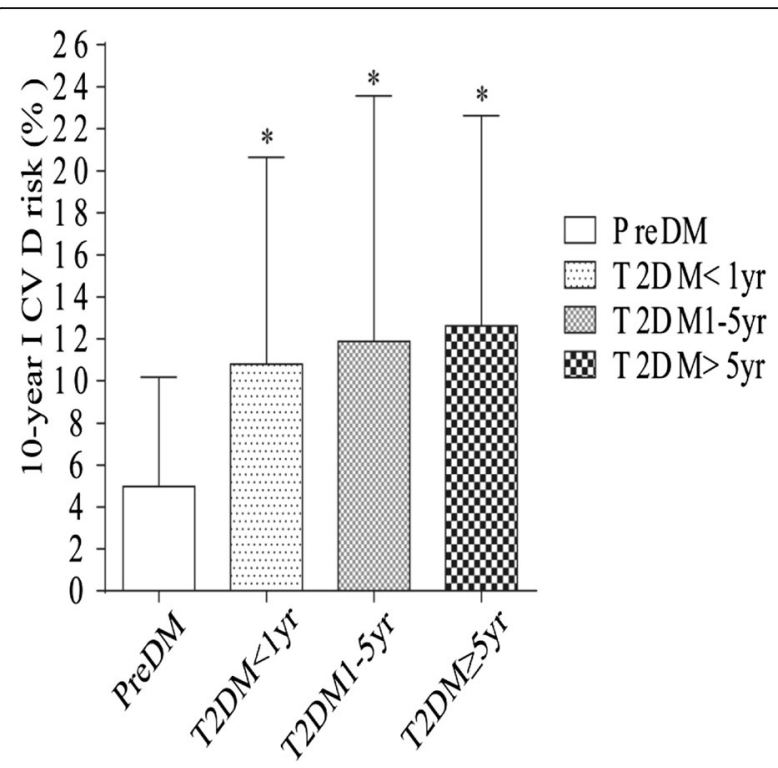

Fig. 2 Assessment of 10-year ICVD risk in females with PreDM and T2DM. Values are mean \pm SD. ${ }^{*} p \leq 0.05$, compared T2DM to PreDM in female patients

year ICVD pattern being worse in females. First, recent accumulating evidence demonstrates that diabetes alters estrogenrelated protective mechanisms and causes pronounced adverse changes in cardiovascular risk factors leading to enhanced atherogenesis in females [27, 28]. In our study, the average age of females with T2DM was about 61 years; gradually decreasing estrogen levels may indirectly result in higher ICVD risk. Second, in a cross-sectional analysis,females were less likely than males to meet the goals for LDL cholesterol; this suggests the need for gender-specific approaches [29]. A similar result, found in this research population, was that females with T2DM had a higher prevalence rate of hypertension and hyperlipidemia. Additionally, females with T2DM compared to males had significantly less optimal values for HOMA-IR. Insulin resistance levels are important and should be highlighted because insulin resistenace has been shown to be an independent risk factor for cardiovascular events [30].

Hypertension and hyperlipidemia are well-established causal risk factors for ischemic cardiovascular disease [31]. In the current study, the prevalence, awareness, treatment and control of hypertension overall were $66.44 \%, 78.60 \%, 65.38 \%$ and $31.10 \%$, respectively. More than half of the T2DM patients had hypertension. A similar result was obtained in a US study that found that the frequency of hypertension was
63\% in 2012 among both prevalent and newly-diagnosed T2DM cohorts [32]. Although the awareness and treatment rate of hypertension in our study signals a need for more interventions in both males and females, it is on the control rate that researchers and clinicians in China must first focus. In addition, it should be noted that encouraging primary care physicians and other public healthcare professionals to continue to expand their efforts to control high blood pressure is necessary for Chinese patients. We also observed that in the current study, hyperlipidemia rates of prevalence, awareness, treatment and control were $57.56 \%, 29.15 \%, 8.30 \%$ and $3.47 \%$, respectively. A similar result on prevalence was found in a US study; it reported that the frequency of hyperlipidemia with T2DM was 56.9\% [32].The awareness, treatment and control of hyperlipidemia were not at high levels, possibly suggesting that people do not pay attention to their health or people with hyperlipidemia do not adhere to prescribed treatments, even after becoming aware of hyperlipidemia. Therefore, it is apparent that a national hyperlipidemia education program to promote community- and clinicbased serum lipid screening is urgently needed in China.

The strength of the current study is that this was the first study to report the absolute 10-year ICVD risk assessment in Chinese patients with PreDM or T2DM. The current study has several limitations. First, the current study lacked data on the awareness, treatment and control of hypertension and hyperlipidemia in those with PreDM, and it also lacked data on physical activity, sedentary behavior time and specific medical treatments, which are strongly associated with the development of the metabolic syndrome, T2DM and CVD. Second, data on smoking, hypertension and hyperlipidemia were self-reported.

\section{Conclusions}

In conclusion, the current study demonstrated that different cut-off points exist for focusing on ischemic cardiovascular diseases: before 5 years for males and less than 1 year for females with T2DM. There is a greater need for cardiovascular risk reduction programs for females with T2DM, as soon as they are diagnosed. Also, given the higher prevalence of hypertension and hyperlipidemia in females, significant resources focused on them must be expended. Diabetes education in males is also needed before 5 years to be able to improve awareness, treatment and control of hypertension and hyperlipidemia, in particular. A key to reducing 10-year risk is continued intensive follow-up, which may or may not be possible in China with so many Chinese affected.

Table 2 Prevalence, awareness, treatment and control of hypertension and hyperlipidemia in T2DM patients according to sex $(n=900)$

\begin{tabular}{|c|c|c|c|c|c|c|c|c|c|c|c|c|}
\hline \multirow[t]{2}{*}{ Risk factor } & \multicolumn{2}{|c|}{ Prevalence n (\%) } & \multirow{2}{*}{$\begin{array}{l}p \\
\text { value }\end{array}$} & \multicolumn{2}{|c|}{ Awareness n (\%) } & \multirow{2}{*}{$\begin{array}{l}p \\
\text { value }\end{array}$} & \multicolumn{2}{|c|}{ Treatment n (\%) } & \multirow{2}{*}{$\begin{array}{l}P \\
\text { value }\end{array}$} & \multicolumn{2}{|c|}{ Control n (\%) } & \multirow{2}{*}{$\begin{array}{l}p \\
\text { value }\end{array}$} \\
\hline & Male & Female & & Male & Female & & Male & Female & & Male & Female & \\
\hline Hypertension & 197(21.89) & $401(44.56)$ & $<0.001$ & $163(27.26)$ & 307(51.34) & $<0.001$ & $132(22.07)$ & 259(43.31) & $<0.001$ & $65(10.87)$ & $121(20.23)$ & $<0.00$ \\
\hline Hyperlipidemia & 158(17.56) & $360(40.00)$ & $<0.001$ & $60(11.58)$ & $91(17.57)$ & 0.006 & $11(2.12)$ & $32(6.18)$ & 0.001 & $7(1.35)$ & $11(2.12)$ & 0.3 \\
\hline
\end{tabular}




\section{Appendix}

Evaluation sheet for estimating 10-year risk of ischemic cardiovascular disease incidence for males and females
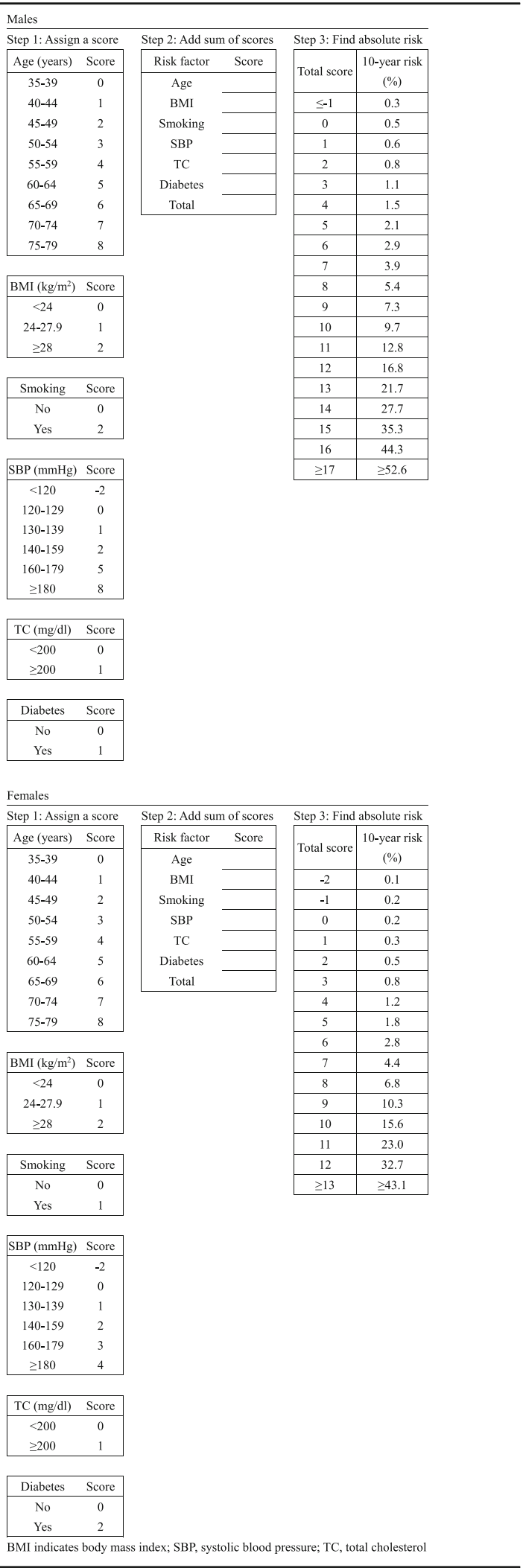

\section{Abbreviations}

2hPG: 2-h plasma glucose; BMI: Body mass index; DBP: Diastolic blood pressure; FPG: Fasting plasma glucose; FSI: Fasting serum insulin; HbA1c: Glycated hemoglobin; HDL: High density lipoproteins; HOMAIR: Homeostasis model assessment of insulin resistance; HOMA$\beta$ : Homeostasis model assessment of beta cell function; LDL: Low density lipoproteins; SBP: Systolic blood pressure; TC: Total cholesterol; TG: Triglyceride; WC: Waist circumference

\section{Acknowledgements}

Not applicable.

\section{Authors' contributions}

QQL and YCX provided funding and administrative support and participated in the design of the study. LHY contributed in drafting of the manuscript. AF revised, critically, important intellectual content before the manuscript was submitted. YYZ, XDY, JNL, and XYW recruited the participants and took responsibility for data collection. $L Y, Z M J$, and $C L$ performed data analysis. All authors read and approved the final manuscript.

\section{Funding}

This work was supported by grants from Research Project of State Administration of Traditional Chinese Medicine of the People's Republic of China (JDZX2015132) and Jiangsu Provincial Personnel Beauro (2014-WSN025). The funding bodies had no role in the design of the study and collection, analysis, and interpretation of data and in writing the manuscript.

\section{Availability of data and materials}

The datasets used and/or analysed during the current study are available from the corresponding author on reasonable request.

\section{Ethics approval and consent to participate}

The study was performed in accordance with the Declaration of Helsinki and approved by the Ethics Committees of Jiangsu Province Academy of Traditional Chinese Medicine, Affiliated Hospital of Henan University of Science and Technology, Shanxi Provincial People's Hospital and West China Hospital. Written informed consent from all participants was obtained.

\section{Consent for publication}

Not applicable.

\section{Competing interests}

The authors declare that they have no competing interests.

\section{Author details}

'Department of Health Education, Affiliated Hospital of Integrated Traditional Chinese and Western Medicine, Nanjing University of Chinese Medicine, \#100 Hongshan Road, Qi xia District, Nanjing, Jiangsu Province 210028, China.

${ }^{2}$ Jiangsu Province Academy of Traditional Chinese Medicine, 100 Shizi Street, Hongshan Rd, Qi Xia District, Nanjing, Jiangsu Province, China. ${ }^{3}$ Eastern Branch of Suzhou Municipal Hospital, 16 Baita West Rd, Gu Su District, Suzhou, Jiangsu Province, China. ${ }^{4}$ College of Nursing (ISP Program), University of Missouri-St. Louis, One University Blvd, M/C 529, St. Louis, MO, USA. ${ }^{5}$ Nursing College, Nanjing University of Chinese Medicine, 138 Xianlin Rd, Qi Xia District, Nanjing, Jiangsu Province, China. ${ }^{6}$ Department of Endocrinology, Shanxi Provincial People's Hospital, 29 Twin Towers Temple Street, Taiyuan, Shanxi Province, China. ${ }^{7}$ Department of Endocrinology, West China Hospital, West China School of Medicine, Sichuan University, 37 Guoxue Lane, Wu Hou District, Chengdu, Sichuan Province, China.

${ }^{8}$ Department of Endocrinology, Affiliated Hospital of Henan University of Science and Technology, 24 Jinghua Rd, Luoyang, Henan Province, China. 'Hainan People's Hospital-Longhua, \#8 Longhua Rd, Longhua District Hainan, Haikou 570102, China.

Received: 11 September 2018 Accepted: 23 October 2019

Published online: 18 December 2019

\section{References}

1. International Diabetes Federation. IDF Diabetes Atlas 8th Edition.

2. Xu Y, Wang LM, He J, Bi YF, Li M, Wang TG, et al. Prevalence and control of diabetes in Chinese adults. JAMA. 2013;310(9):948-58. 
3. Yang WY, Lu JM, Weng JP, Jia WP, Ji LN, Xiao JZ, et al. Prevalence of diabetes among men and women in China. N Engl J Med. 2010;362(25): 1090-101.

4. Li G, Zhang P, Wang J, Gregg EW, Yang W, Gong Q, et al. The long-term effect of lifestyle interventions to prevent diabetes in the China Da Qing diabetes prevention study: a 20-year follow-up study. Lancet. 2008; 371(9626):1783-9.

5. Levitzky YS, Pencina MJ, D'Agostino RB, Meigs JB, Murabito JM, Vasan RS, et al. Impact of impaired fasting glucose on cardiovascular disease. J Am Coll Cardiol. 2008;51(3):264-70.

6. Roth GA, Johnson C, Abajobir A, Abd-Allah F, Abera SF, Abyu G, et al. Global, regional, and National Burden of cardiovascular diseases for 10 causes, 1990 to 2015. J Am Coll Cardiol. 2017;70(1):1-25.

7. Zhou M, Wang H, Zhu J, Chen W, Wang L, Liu S, et al. Cause-specific mortality for 240 causes in China during 1990-2013: a systematic subnational analysis for the global burden of disease study 2013. Lancet. 2016:387(10015):251-72.

8. National Cardiovascular Center. Chinese cardiovascular disease report 2016[M]. Beijing: Encyclopedia of China Publishing House; 2017.

9. Peters SA, Huxley RR, Sattar N, Woodward M. Sex differences in the excess risk of cardiovascular diseases associated with type 2 diabetes: potential explanations and clinical implications. Curr Cardiovasc Risk Rep. 2015;9(7):36.

10. Barrett-Connor EL, Cohn BA, Wingard DL, Edelstein SL. Why is diabetes mellitus a stronger risk factor for fatal ischemic heart disease in women than in men? The Rancho Bernardo Study JAMA. 1991;265:627-31.

11. Towfighi A, Zheng $L$, Ovbiagele B. Sex-specific trends in midlife coronary heart disease risk and prevalence. Arch Intern Med. 2009;169(19):1762-6.

12. Task group on the study of the comprehensive risk assessment and intervention program for coronary heart disease and stroke in the national "fifteen" project. Assessment of risk of ischemic cardiovascular disease in Chinese. Chin J Cardiol. 2003; 31(12):893-901.

13. Ma X, Bai X, Karmacharya UK, Huang D, Huang Y, Xie X, et al. Cardiovascular risks in Kazakh population in Xinjiang Province of China. Ethn Dis. 2014; 24(3):316-20.

14. Wu LX, Zen Q, Qiang DC. The distribution of 10-year cardiovascular risk in Chinese adults: analysis of the China health examination database (CHED) 2008. J Geriatr Cardiol. 2010;7(3):131-7.

15. Xu Q, Shen JY, Shi R, Zhao H. Ten year risk assessment of ischemic cardiovascular disease and intervention analysis among middle-aged residents with moderate risk and above in a Shanghai-based community. Fam Med Comm Health. 2017;5(1):92-100.

16. Diabetes Association of Chinese Medical Association. Guidelines for the prevention and treatment of type 2 diabetes mellitus in China (2013 edition). Chinese Journal of diabetes. 2014;6(7):447-98.

17. National "fifteen" scientific and technological research group. Development and evaluation of risk assessment methods for ischemic cardiovascular diseases in Chinese people. Chin J Cardiovasc Dis 2003; 31 (12): 893-901.

18. Chinese Medical Association Diabetes Branch. China type 2 diabetes prevention and control guide (2013 edition). CJDM. 2014;7(6):448-96.

19. Petrie JC, O'Brien ET, Littler WA, De SM. Recommendations on blood pressure measurement. Br Med J. 1986;293(6547):611-5.

20. Liu LS, Wu ZS, Zhu DL. Guidelines for hypertension prevention and treatment in China (Third Edition). 2010

21. Gu DF, Jiang $H, W u X G$. Prevalence, awareness, treatment and control of hypertension in Chinese adults. Chin J Prevent Med. 2003:37(2):84.

22. Joint Committee on the development of guidelines for the prevention and control of dyslipidemia in Chinese adults. Guidelines for the prevention and treatment of dyslipidemia in Chinese adults. Chin J Cardiol. 2007; 35(5):390-419.

23. Matthews DR, Hosker JP, Rudenski AS, Naylor BA, Treacher DF, Turner RC. Homeostasis model assessment: insulin resistance and beta-cell function from fasting plasma glucose and insulin concentrations in man. Diabetologia. 1985;28:412-9.

24. Chinese Physician Association. Cardiovascular Department of Internal Medicine, Chinese journal of internal medicine editorial committee, Chinese expert consensus about primary prevention of cardiovascular disease. Chinese J Dep Intern Med. 2010;49(2):174-85.

25. Anagnostis P, Majeed A, Johnston DG, Godsland IF. Cardiovascular risk in women with type 2 diabetes mellitus and prediabetes: is it indeed higher than men? Eur J Endocrinol. 2014;171(6):R245-55.

26. Ezenwaka CE, Nwagbara E, Seales D, Okali F, Hussaini S, Raja B, et al. Prediction of 10-year coronary heart disease risk in Caribbean type 2 diabetic patients using the UKPDS risk engine. Int J Cardiol. 2009;132(3): 348-53.

27. Steinberg HO, Paradisi G, Cronin J, Crowde K, Hempfling A, Hook G, et al. Type II diabetes abrogates sex differences in endothelial function in premenopausal women. Circulation. 2000;101(17):2040-6.

28. Juutilainen A, Kortelainen S, Lehto S, Rönnemaa T, Pyörälä K, Laakso M. Gender difference in the impact of type 2 diabetes on coronary heart disease risk. Diabetes Care. 2004;27(12):2898-904.

29. Wexler DJ, Grant RW, Meigs JB, Nathan DM, Cagliero E. Sex disparities in treatment of cardiac risk factors in patients with type 2 diabetes. Diabetes Care. 2005;28(3):514-20.

30. Wang CM, Li F, Guo JJ, Li CC, Xu DS, Wang B. Insulin resistance, blood glucose and inflammatory cytokine levels are risk factors for cardiovascular events in diabetic patients complicated with coronary heart disease. Experiment Therapeutic Med. 2018;15(2):1515-9.

31. Piepoli MF, Hoes AW, Agewall S, Albus C, Brotons C, Catapano AL. 2016 European guidelines on cardiovascular disease prevention in clinical practice. The sixth joint task force of the European Society of Cardiology and other societies on cardiovascular disease prevention in clinical practice. Eur J Prev Cardiol. 2016;23(11):NP1-96.

32. Weng W, Liang Y, Kimball ES, Hobbs T, Kong SX, Sakurada B, et al. Decreasing incidence of type 2 diabetes mellitus in the United States, 2007-2012: epidemiologic findings from a large US claims database. Diabetes Res Clin Pract. 2016;117:111-8.

\section{Publisher's Note}

Springer Nature remains neutral with regard to jurisdictional claims in published maps and institutional affiliations.
Ready to submit your research? Choose BMC and benefit from:

- fast, convenient online submission

- thorough peer review by experienced researchers in your field

- rapid publication on acceptance

- support for research data, including large and complex data types

- gold Open Access which fosters wider collaboration and increased citations

- maximum visibility for your research: over $100 \mathrm{M}$ website views per year

At $\mathrm{BMC}$, research is always in progress.

Learn more biomedcentral.com/submissions 